Case report: Scalp Block with dexmedetomidine as an additive to local anesthetics for Basal Cell Carcinoma (BCC) surgery to a high risk patient

D. Zosimidis ${ }^{1}$, E. Koraki ${ }^{1}$, Z. Stergiouda ${ }^{1}$, A. Gkiouliava1 ${ }^{1}$, M. Arapidou ${ }^{1}$, A. Trikoupi ${ }^{1}$ 1Department of Anesthesiology, Georgios Papanikolaou General Hospital of Thessaloniki - Thessaloniki (Greece)

-sedation, anxiolysis and optimal analgesia - without disturbing hemodynamic status

$95 \mathrm{y}$ old

COPD, CAD, paroxysmal AF,

HTN, monocular, BPH, METS<3
Sensory block was

established at 10 minutes The 180 min surgery was carried out under mild to moderate sedation with the patient hemodynamic stable and no complaint of pain or dissatisfaction He was discharged from PACU with an Aldrete Score of 9. Analgesia endured 20 hours and no signs of delirium occurred

\title{
Discussion:
}

Scalp block can be used as an adjunct to general anesthesia or serve as the main anesthetic method.

Dexmedetomidine when mixed with a LA

$\checkmark$ can prolong duration of block

$\checkmark$ can provide optimal balance between block features and systemic effects while avoiding adverse events

High risk patients can be managed as a day case

\section{Learning points:}

Scalp block is safe and cost effective

Dexmedetomidine may serve as an asset that offers sedation without respiratory depression
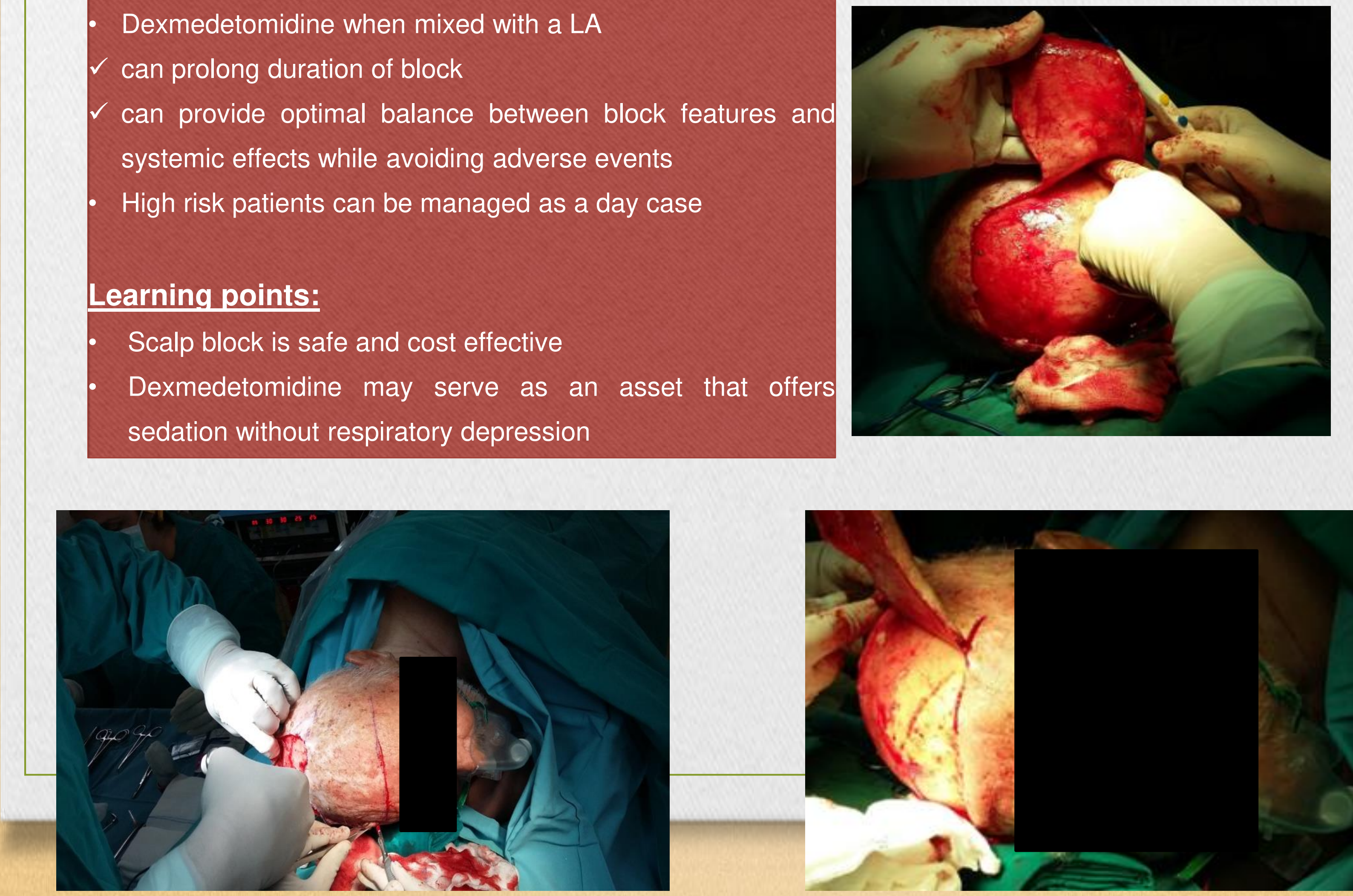